\title{
A Novel Method for Characterization of Peripheral Nerve Fiber Size Distributions by Group Delay Measurements and Simulated Annealing Optimization
}

\author{
Robert B. Szlavik and Galen E. Turner III
}

\begin{abstract}
The ability to determine the characteristics of peripheral nerve fiber size distributions would provide additional information to clinicians for the diagnosis of specific pathologies of the peripheral nervous system. Investigation of these conditions, using electro-diagnostic techniques, is advantageous in the sense that such techniques tend to be minimally invasive yet provide valuable diagnostic information. One of the principal electro-diagnostic tools available to the clinician is the nerve conduction velocity test. While the peripheral nerve conduction velocity test can provide useful information to the clinician regarding the viability of the nerve under study, it is a single parameter test that yields no detailed information about the characteristics of the functioning nerve fibers within the nerve trunk. In this study we present a technique based on a decomposition of the maximal compound evoked potential and subsequent determination of the group delay of the contributing nerve fibers. The fiber group delay is then utilized as an initial estimation of the nerve fiber size distribution and the concomitant temporal propagation delays of the associated single fiber evoked potentials to a reference electrode. Subsequently the estimated single fiber evoked potentials are optimized against the template maximal compound evoked potential using a simulated annealing algorithm. Simulation studies, based on deterministic single fiber action potential functions, are used to demonstrate the robustness of the proposed technique in the presence of noise associated with variations in distance between the nerve fibers and the recording electrodes between the two recording sites.
\end{abstract}

\section{INTRODUCTION}

$\mathrm{T}$ he nerve conduction velocity test provides clinically useful information in the diagnosis of peripheral neuropathies, such as Carpal Tunnel Syndrome [1;2]. Since nerve conduction velocity studies are essentially single parameter measurements of the gross conduction properties of the underlying nerve trunk, such studies are not suited to providing detailed information regarding the characteristics of the underlying nerve fibers that contribute to the compound evoked potential.

A more robust measurement technique would involve the ability to extract information about the viability of the

Manuscript received April 7, 2008

This work was supported in part by the Department of the Navy, Office of Naval Research.

R. B. Szlavik is with the Department of Biomedical \& General Engineering, California Polytechnic State University, San Luis Obispo, CA 93407-0350 USA (phone: 805-756-2025; fax: 805-756-6424; e-mail: rszlavik@calpoly.edu).

G. E. Turner III is with the College of Engineering and Science, Louisiana Tech University, Ruston, LA 71272-0046 USA (phone: 318257-2582; e-mail: gturner@latech.edu). underlying nerve fibers which could potentially provide useful information to the clinician. As an example, information related to the size distribution of contributing nerve fibers can be used to differentiate between different clinical conditions such as Chronic Inflammatory Demyelinating Polyneuropathy, which selectively impacts larger nerve fibers, or Early Diabetic Peripheral Neuropathy, which impacts smaller fibers $[3 ; 4]$.

There is a large body of literature devoted to describing various techniques for determining the nerve fiber conduction velocity distribution (CVD). The pioneering work of Cummins and Dorfman [5;6] describe techniques that use two compound action potentials to estimate the conduction velocity distribution using a least squares approach. Common to these studies was the assumption that fibers included in a specific velocity class have identical evoked potential waveforms.

More recently there have been several additional studies including the work of Gonzalez-Cueto, Papadopoulou and Gu [7-9]. The study presented by $\mathrm{Tu}$ et. al. focused on a regularized least squares algorithm but features many of the same assumptions associated with waveform commonality related to velocity classes that were made in earlier work [10]. This study also investigated the impact of noise on the integrity of the estimated CVD.

In this paper, we discuss a novel technique, presented previously by the author [11], for estimating the size distribution of contributing nerve fibers which is linearly related to the CVD. The technique is based on an estimation of the group delay between two sets of recording electrodes associated with the individual fibers that contribute to a maximal compound evoked potential. The group delay information is then used to estimate the diameters of the activated fibers as well as the propagation delays of individual single fiber evoked potentials to a reference electrode. This process allows for reconstruction of an estimated maximal compound evoked potential, from the individual single fiber evoked potentials, at the first recording site. The previously presented group delay technique is expanded upon in this paper by introduction of a simulated annealing optimization algorithm. This algorithm is used to vary the diameter and concomitant propagation delays associated with the estimated single fiber evoked potential waveforms to search for an improved fit with the template maximal compound evoked potential from the same recording site.

The basic methodology behind the technique is presented by utilizing a closed form mathematical model of a single 
fiber evoked potential waveform that allows us to demonstrate the robustness of the technique under noisy conditions introduced by random variations in the perpendicular distance between the fiber and recording electrode [12].

\section{METHOD}

The simulation for determination of the group delay is premised on the physical setup shown in Figure 1, where a stimulator is used to excite a subcutaneous nerve trunk consisting of a group of electrically independent nerve fibers. The propagating compound evoked potential is detected at two recording sites. Using a series of successively increasing current stimulus pulses, the successively recorded compound evoked potentials can be decomposed into their constituent single fiber action potentials in a manner analogous to the protocol used in the McComas et al. motor unit number estimation technique [13].

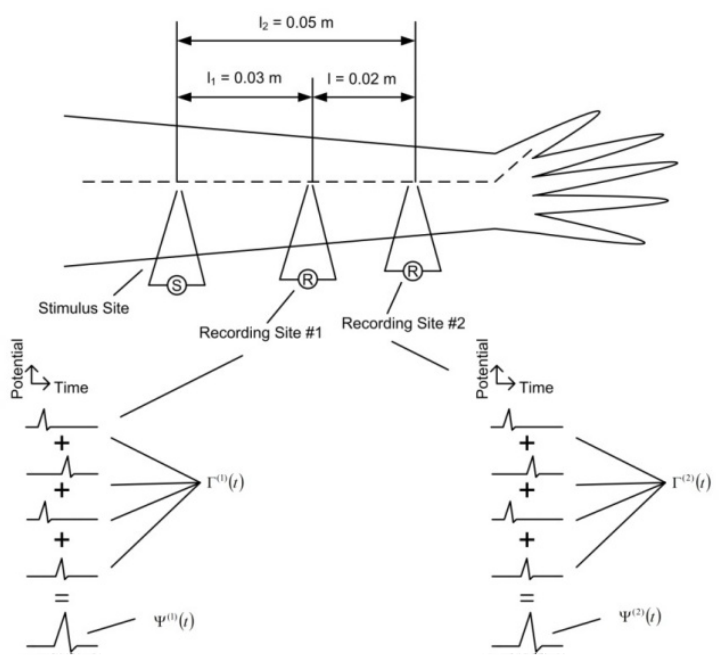

Figure 1. Conceptual physical configuration of the proposed method. The diagram shows the stimulus and recording sites as well as the relationship between the individual single fiber evoked potentials sets $\boldsymbol{\Gamma}^{(\mathbf{1})}(\boldsymbol{t})$ and $\boldsymbol{\Gamma}^{(2)}(\boldsymbol{t})$ to the maximal compound evoked potentials $\Psi^{(1)}(t)$ and $\Psi^{(2)}(t)$. The figure is not drawn to scale and is indicative of stimulation and recording sites at convenient locations along the median nerve. From an experimental perspective, implementation could involve stimulation of the median nerve at the anterior cubital fossa with recording sites placed more distally at anatomically convenient locations such as the wrist. This type of placement would result in distances that are larger than those indicated on the figure.

An empirically determined nerve fiber diameter distribution [14] was used to generate a random population of one hundred nerve fiber diameters using a technique described by Szlavik and de Bruin [15]. The distribution in (1) was used to generate the fiber diameter population.

$$
p_{d}\left(d_{k}\right)=\sum_{h=1}^{4} \frac{\beta_{h}}{\sigma_{h} \sqrt{2 \pi}} \exp \left[-\frac{\left(d_{k}-\mu_{h}\right)^{2}}{2 \sigma_{h}^{2}}\right]
$$

The parameters shown in Table 1 were used in the distribution of (1).

TABLE I

PARAMETER VALUES USED IN THE FIBER DIAMETER DISTRIBUTION

\begin{tabular}{lll}
\hline \hline Symbol & \multicolumn{1}{c}{ Quantity } & \multicolumn{1}{c}{ Value } \\
\hline$\beta_{1}$ & complete distribution $1^{\text {st }}$ mode scaling const. & $0.05(\mathrm{~m})$ \\
$\sigma_{1}$ & complete distribution $1^{\text {st }}$ mode std. dev. & $0.1274(\mu \mathrm{m})$ \\
$\mu_{1}$ & complete distribution $1^{\text {st }}$ mode mean & $0.5(\mu \mathrm{m})$ \\
$\beta_{2}$ & complete distribution $2^{\text {nd }}$ mode scaling const & $0.25(\mathrm{~m})$ \\
$\sigma_{2}$ & complete distribution $2^{\text {nd }}$ mode std. dev. & $0.8493(\mu \mathrm{m})$ \\
$\mu_{2}$ & complete distribution $2^{\text {nd }}$ mode mean & $3(\mu \mathrm{m})$ \\
$\beta_{3}$ & complete distribution $3^{\text {rd }}$ mode scaling const. & $0.3(\mathrm{~m})$ \\
$\sigma_{3}$ & complete distribution $3^{\text {rd }}$ mode std. dev. & $1.699(\mu \mathrm{m})$ \\
$\mu_{3}$ & complete distribution $3^{\text {rd }}$ mode mean & $7.5(\mu \mathrm{m})$ \\
$\beta_{4}$ & complete distribution $4^{\text {th }}$ mode scaling const. & $0.4(\mathrm{~m})$ \\
$\sigma_{4}$ & complete distribution $4^{\text {th }}$ mode std. dev. & $1.699(\mu \mathrm{m})$ \\
$\mu_{4}$ & complete distribution $4^{\text {th }}$ mode mean & $13(\mu \mathrm{m})$ \\
& & \\
\hline \hline
\end{tabular}

Fiber diameters less than $5 \mu \mathrm{m}$ were excluded from the population yielding a sub-population of $m=60$ fibers. The maximum nerve fiber diameter in the distribution was 17.1 $\mu \mathrm{m}$. This randomly generated fiber diameter distribution formed the template distribution population $\boldsymbol{d}$.

The population of nerve fibers was subjected to a series of virtual stimulus pulses of successively increasing current amplitude where $\Omega_{i}$ is the amplitude of the stimulus current pulse at each increment $i$. An activation function $\xi(d)$ is used to determine whether a given stimulus current amplitude was sufficient to excite each fiber with diameter $d$ as per (2) where $\zeta=10 \mathrm{~mA}$ and $\eta=3.5 \times 10^{5} \mathrm{~m}^{-1}$.

$$
\xi(d)=\zeta \exp [-\eta d]
$$

For each recording site $n=1,2$, the compound evoked potential $\Psi_{i}^{(n)}(t)$ is computed for each increment $i$ of the stimulus current amplitude as per (3).

$$
\Psi_{i}^{(n)}(t)=\sum_{k=1}^{m} u\left[\Omega_{i}-\xi\left(d_{k}\right)\right] G\left[v_{k} \cdot\left(t-\delta_{k}^{(n)}\right), \bar{r}\right]
$$

In (3), the single fiber action potential waveform $G\left[v_{k}\right.$. $\left.\left(t-\delta_{k}^{(n)}\right), \bar{r}\right]$ contributes to the compound evoked potential if the argument of the step function $u$ is positive where $t$ is the time in seconds, $v_{k}$ is the conduction velocity of the $k^{\text {th }}$ fiber, $\delta_{k}^{(n)}$ is the propagation delay, in seconds, of the single fiber action potential from the stimulus site to the $n^{\text {th }}$ recording site and $\bar{r}$ is the perpendicular depth between the recording site and the center of the $k^{\text {th }}$ fiber.

The function $G$ is the normalized model of the single fiber action potential proposed by Fleisher et. al. where the function has been normalized to the current through the second pole such that $G=g / I$ as per (4). All other parameters are as described in Fleisher [12] and were assigned values $a_{k}=d_{k} / 2, \quad s_{k}=5 \cdot a_{k}, \quad \bar{r}=1 \mathrm{~mm}$, $v_{k}=c \cdot d_{k}, \quad \alpha=0.75, \quad \sigma_{e}=1.0 \mathrm{~S} / \mathrm{m} \quad$ and $D_{k}=$ $\left(a_{k}+s_{k}\right) /\left(\bar{r}+s_{k}\right)$. 


$$
\begin{aligned}
& G\left[v_{k} \cdot\left(t-\delta_{k}^{(n)}\right), \bar{r}\right] \\
& =\frac{D_{k}^{2}}{4 \pi \sigma_{e} a_{k}}\left[\alpha \exp \left\{-\left(\frac{D_{k}}{4}\right)^{2}\left(\frac{v_{k} \cdot\left(t-\delta_{k}^{(n)}\right)+s}{a_{k}}\right)^{2}\right\}\right. \\
& -\exp \left\{-\left(\frac{D_{k}}{4}\right)^{2}\left(\frac{v_{k} \cdot\left(t-\delta_{k}^{(n)}\right)-s}{a_{k}}\right)^{2}\right\} \\
& \left.+(1-\alpha) \exp \left\{-\left(\frac{D_{k}}{4}\right)^{2}\left(\frac{v_{k} \cdot\left(t-\delta_{k}^{(n)}\right)-u}{a_{k}}\right)^{2}\right\}\right]
\end{aligned}
$$

After the compound evoked potentials are computed for each virtual current step $\Omega_{i}$, the series of compound evoked potentials at each recording site $\boldsymbol{\Psi}^{(\mathbf{1})}(\boldsymbol{t})$ and $\boldsymbol{\Psi}^{(\mathbf{2})}(\boldsymbol{t})$ are decomposed into a series of waveforms that nominally consist of the contributing single fiber action potentials at each simulated current step $\boldsymbol{\Gamma}^{(\mathbf{1})}(\boldsymbol{t})$ and $\boldsymbol{\Gamma}^{(\mathbf{2})}(\boldsymbol{t})$ as per (5).

$$
\Gamma_{i-1}^{(n)}(t)=\Psi_{i}^{(\mathrm{n})}(\mathrm{t})-\Psi_{\mathrm{i}-1}^{(\mathrm{n})}(\mathrm{t}) \quad \text { for } 2 \leq \mathrm{i} \leq \mathrm{q}+1
$$

If the current steps are small enough, then the waveforms $\Gamma^{(n)}(\boldsymbol{t})$ will consist of individual contributing single fiber action potentials or no waveform where a stimulus current increment does not result in an additional recruited fiber. However, a perfect decomposition will not always be achievable due to the finite discretization of the stimulus current steps. Some of the $q$ non-zero waveforms in the set $\Gamma^{(n)}(\boldsymbol{t})$ will consist of more than one single fiber action potential.

Once the decomposition is complete, the individual decomposed waveforms from the two recording sites can be used to compute an estimate of the group delay associated with each contributing nerve fiber where the frequency response of a given fiber $H_{i-1}(f)$ is as shown in (6).

$$
H_{i-1}(f)=\frac{\mathfrak{F}\left[\Gamma_{i-1}^{(2)}(\mathrm{t})\right]}{\mathfrak{F}\left[\Gamma_{i-1}^{(1)}(t)\right]}
$$

The frequency response is computed by dividing the Fourier Transform of the single fiber evoked potential associated with the more distal recording site by the Fourier Transform of the single fiber evoked potential associated with the more proximal recording site. Since each $H_{i-1}(f)=\left|H_{i-1}(f)\right| \angle \Theta_{i-1}(f)$, an estimate of the group delay $\tau_{i-1}$ for each pair of non-zero decomposed waveforms $\Gamma_{i-1}^{(1)}(t)$ and $\Gamma_{i-1}^{(2)}(t)$ can be computed from (7).

$$
\tau_{i-1}=-\frac{1}{2 \pi} \frac{d \Theta_{\mathrm{i}-1}(f)}{d f}
$$

In practice, a least squares line is fitted to the phase response $\Theta_{i-1}(f)$ for the $H_{i-1}(f)$ computed for each pair of non-zero decomposed waveforms $\Gamma_{i-1}^{(1)}(t)$ and $\Gamma_{i-1}^{(2)}(t)$ which facilitates the computation of the associated group delay $\tau_{i-1}$. The estimated group delays for the contributing nerve fibers are used to compute an estimate of the associated fiber diameters from $(8)$ where $l(m)$ is the distance between the two recording sites and $c=3.0 \times 10^{6} \mathrm{~s}^{-1}$.

$$
d_{i-1}=\frac{l}{c \tau_{i-1}}
$$

Once the estimated group delay is computed for each nonzero pair of decomposed waveforms $\Gamma_{i-1}^{(1)}(t)$ and $\Gamma_{i-1}^{(2)}(t)$, an estimate of the sequence of nerve fiber diameters $\widetilde{\boldsymbol{d}}$ is obtained for the contributing fiber population.

The overall process described above is illustrated in the flowchart of Figure 2.

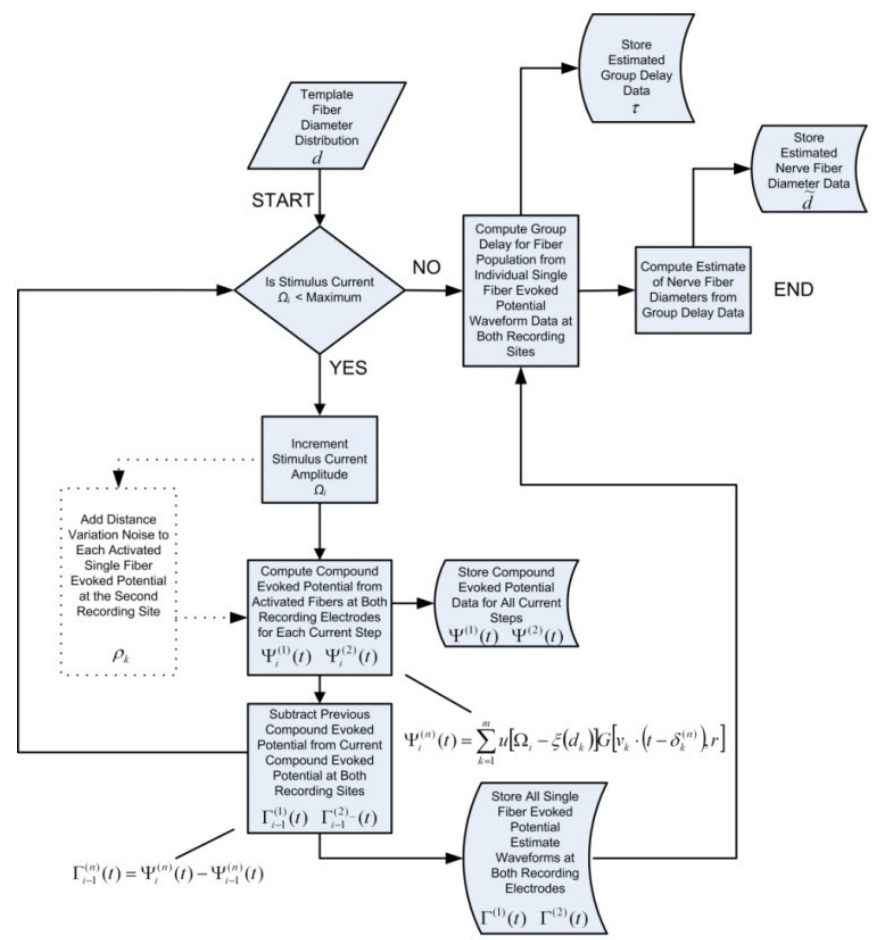

Figure 2. Flowchart of algorithm implemented to calculate an estimate of the group delay of the contributing population of nerve fibers and the estimated fiber diameter set $\widetilde{\boldsymbol{d}}$. The technique is based on determination of an estimate of the group delay associated with each non-zero decomposed waveform $\Gamma_{i-1}^{(1)}(t)$ and $\Gamma_{i-1}^{(2)}(t)$.

Random variations, from the first recording site to the second, in the perpendicular distance between the recording site and the center of the nerve fiber $r$ could reasonably be expected. This characteristic would result in random variations in the form of the contributing single fiber action potentials $G\left[v_{k} \cdot\left(t-\delta_{k}^{(n)}\right), r\right]$ associated with each specific fiber. To simulate the impact of this noise source on the system, $r$ is replaced with a normally distributed random number with a standard deviation of $\rho_{k}$ and a mean of $\bar{r}$. 


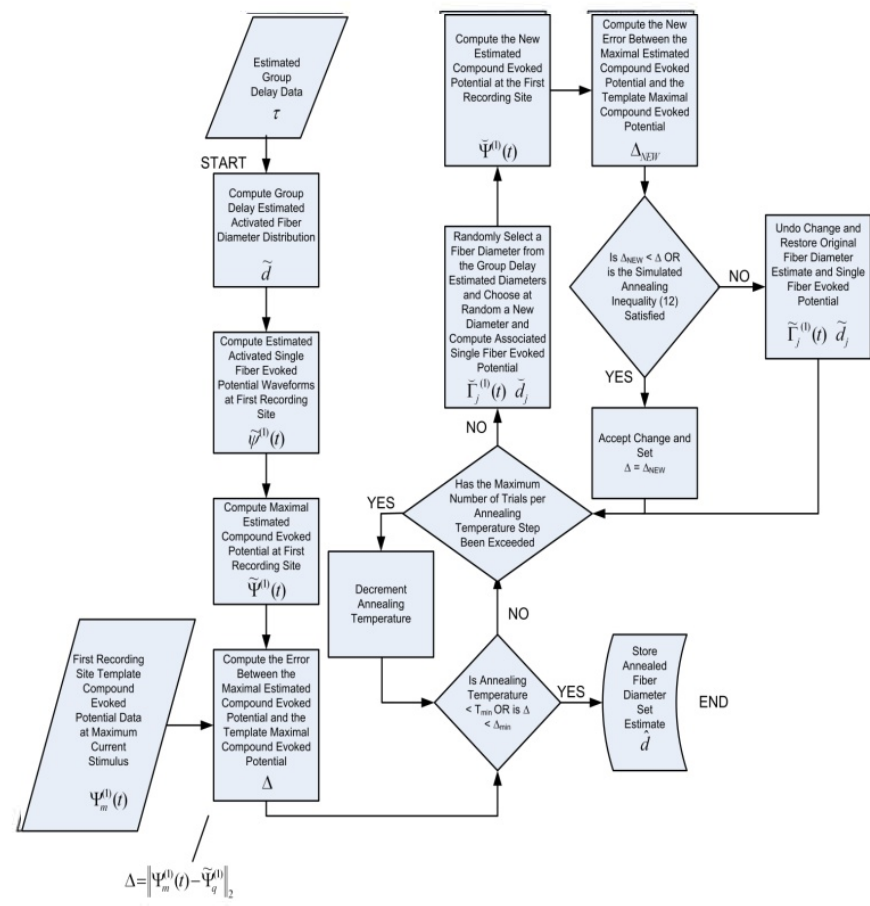

Figure 3. Flowchart of the simulated annealing algorithm that computes an improved estimate of the nerve fiber diameter population set $\widehat{\boldsymbol{d}}$ from the group delay estimated population set $\widetilde{\boldsymbol{d}}$.

The simulated annealing algorithm, first proposed by Metropolis et. al. [16], can be used to compute solutions to NP-complete problems such as the traveling salesman problem, where these solutions are optimal or very close to optimal $[17 ; 18]$. The problem of determining the correct diameters of the contributing nerve fiber population and concomitant forms for each of the single fiber evoked potentials, is also an NP-complete problem to which the simulated annealing algorithm may be applied. The optimized contributing fiber diameter set is a set of diameters $\widehat{\boldsymbol{d}}$ such that (9) is true.

$$
\min \left\|\Psi^{(1)}(t)-\widehat{\Psi}^{(1)}(t)\right\|_{2}
$$

The waveform $\Psi^{(1)}(t)$ is the first recording site template evoked potential at the maximum stimulus current pulse amplitude such that $i=p$ and $\widehat{\Psi}^{(1)}(t)$ is the maximal compound evoked potential estimated from the group delay decomposition at the first recording site as per (10) and optimized using the simulated annealing algorithm. In (10), $q$ is the number of non-zero single fiber evoked potential waveforms obtained through the decomposition shown in (4) and $\tilde{\Gamma}_{j}^{(1)}$ are the decomposed fiber evoked potential waveforms at the first recording site.

$$
\widetilde{\Psi}^{(1)}(t)=\sum_{j=1}^{q} \tilde{\Gamma}_{j}^{(1)}(\mathrm{t})
$$

The algorithm to compute the estimated set of nerve fiber diameters $\widehat{\boldsymbol{d}}$ from simulated annealing is illustrated in the flowchart of Figure 3.
The first step is to compute the group delay estimated set of contributing single fiber action potential waveforms $\widetilde{\boldsymbol{\Gamma}}^{(\mathbf{1})}(\boldsymbol{t})$ and then to compute $\widetilde{\Psi}^{(1)}(t)$ from $(10)$. The error between the template maximal compound evoked potential $\Psi^{(1)}(t)$ and the group delay estimated compound evoked potential with all fibers contributing $\widetilde{\Psi}^{(1)}(t)$ is computed. This error $\Delta$ is formulated in terms of the two-norm difference between the two waveforms.

$$
\Delta=\left\|\Psi^{(1)}(t)-\widetilde{\Psi}^{(1)}(t)\right\|_{2}
$$

The simulated annealing algorithm initially determines whether the $\Delta<\Delta_{\text {MIN }}$ or if the annealing temperature $T<T_{M I N}$. If either of these inequalities hold, the algorithm exits and the optimized set of fiber diameters $\widehat{\boldsymbol{d}}$ is set equal to the set $\widetilde{\boldsymbol{d}}$. In the event neither inequality holds, an estimated fiber diameter $\tilde{d}_{j}$ is randomly chosen from the set $\widetilde{\boldsymbol{d}}$ and a new fiber diameter for this specific fiber is selected at random such that $\tilde{d}_{j}=\breve{d}_{j}$. The concomitant single fiber evoked potential waveform for the randomly generated fiber diameter is computed at the first recording site $\breve{\Gamma}_{j}^{(1)}(t)$ using (4). With this new randomly generated single fiber action potential, a new value is computed for the estimated compound evoked potential associated with the contribution of all fibers in the population $\breve{\Psi}^{(1)}(t)$. Equation (11) is invoked to compute a new error estimate $\Delta_{\mathrm{NEW}}$. If $\Delta_{\mathrm{NEW}}<$ $\Delta$ or if the simulated annealing inequality in (12) holds, where $\Upsilon$ is a uniformly distributed random number between zero and unity, the new randomly generated fiber diameter is accepted.

$$
r \leq \exp \left[-\frac{\left|\Delta-\Delta_{\mathrm{NEW}}\right|}{\mathrm{T}}\right]
$$

If neither of these inequalities hold, the newly generated random fiber diameter $\breve{d}_{j}$ is rejected and replaced with the old fiber diameter $\tilde{d}_{j}$. This process is repeated for the number of trials allowed per annealing step. Once the number of trials is exceeded, the annealing temperature $T$ is reduced and the entire process is repeated for the number of trials allowed per annealing step. The simulated annealing algorithm exits once either $T<T_{M I N}$ or $\Delta<\Delta_{\mathrm{MIN}}$. The output of the algorithm is the annealed estimate of the fiber diameter set $\widehat{\boldsymbol{d}}$.

\section{RESULTS}

A population of 100 randomly generated fibers was utilized in these studies. Fibers with diameters less than 5 $\mu \mathrm{m}$ were rejected yielding a subpopulation of $m=60$ fibers with a maximum diameter of $17.1 \mu \mathrm{m}$. These fibers were subjected to a virtual stimulus pulse train of successively increasing amplitudes ranging from zero to a maximum of 1 $\mathrm{mA}$ in $500 \mathrm{nA}$ steps. At each step the compound evoked potential at both virtual recording sites was computed as per (3) and subsequently, the estimate of single fiber action potential waveforms were obtained at each recording site as 
per (5). The concomitant group delays between the two virtual recording sites were computed yielding the group delay estimated set of fiber diameters $\widetilde{\boldsymbol{d}}$.

A histogram comparing the actual template fiber population $\boldsymbol{d}$ to the group delay estimated population $\widetilde{\boldsymbol{d}}$ is shown in Figure 4.

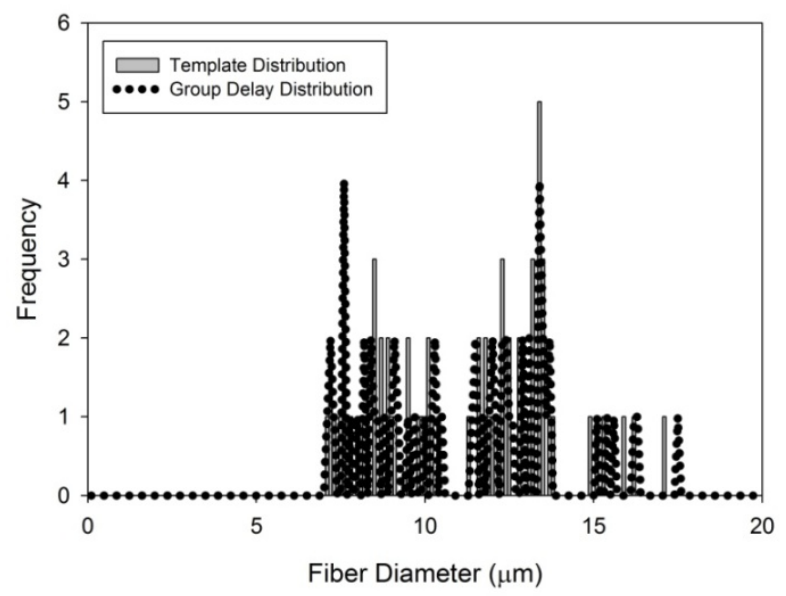

Figure 4. Histogram of template nerve fiber size population $\boldsymbol{d}$ and group delay estimated nerve fiber size population $\widetilde{\boldsymbol{d}}$. The simulation was carried out with an SNR of $20 \mathrm{~dB}$ with respect to random variations in the perpendicular distance between the nerve fiber and the recording site. Chi Square Test results for the two distributions yielded $Q\left(\chi^{2} \mid x\right)=0.7101$.

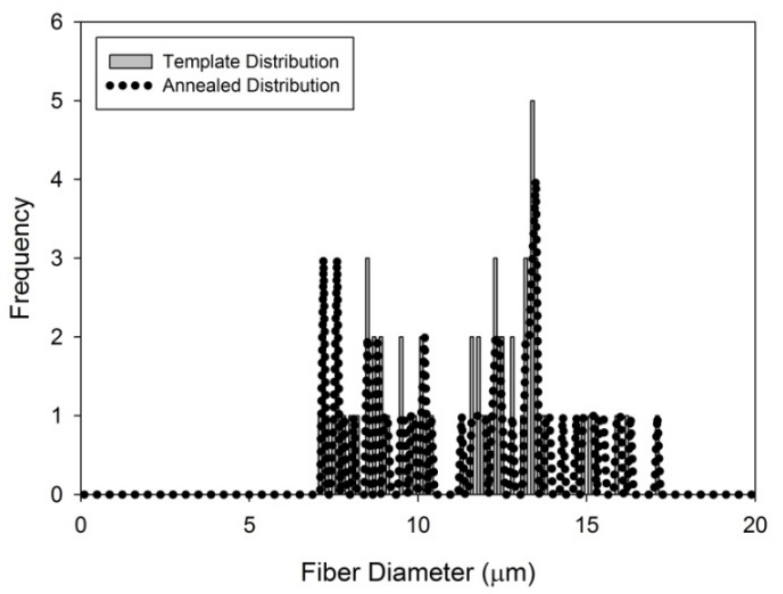

Figure 5. Histogram of the template nerve fiber size population $\boldsymbol{d}$ and annealed nerve fiber size population $\widehat{\boldsymbol{d}}$ from the group delay estimated distribution $\widetilde{\boldsymbol{d}}$ shown in Figure 4. The simulation was carried out with an SNR of $20 \mathrm{~dB}$ with respect to random variations in the perpendicular distance between the nerve fiber and the recording site. Chi Square Test results for the two distributions yielded $Q\left(\chi^{2} \mid x\right)=0.9912$.

The estimated fiber diameter population $\widetilde{\boldsymbol{d}}$ was then optimized using the simulated annealing algorithm described earlier. An initial annealing temperature of $T=10$ was used with an annealing step factor of 0.9 . The minimum annealing temperature was $T_{M I N}=1 \times 10^{-5}$ with a minimum error bound $\Delta_{M I N}=1$. The maximum number of trials for each annealing temperature step was limited to 1000. A histogram comparing the actual template fiber diameter population $\boldsymbol{d}$ to the optimized diameter population $\widehat{\boldsymbol{d}}$ is shown in Figure 5.

Figure 6 compares the maximal template compound evoked potential at the first electrode $\Psi^{(1)}(t)$ with the maximal group delay estimated compound evoked potential $\widetilde{\Psi}^{(1)}(t)$ and the maximal annealed compound evoked potential $\widehat{\Psi}^{(1)}(t)$ for the distributions shown in Figures 4 and 5 .
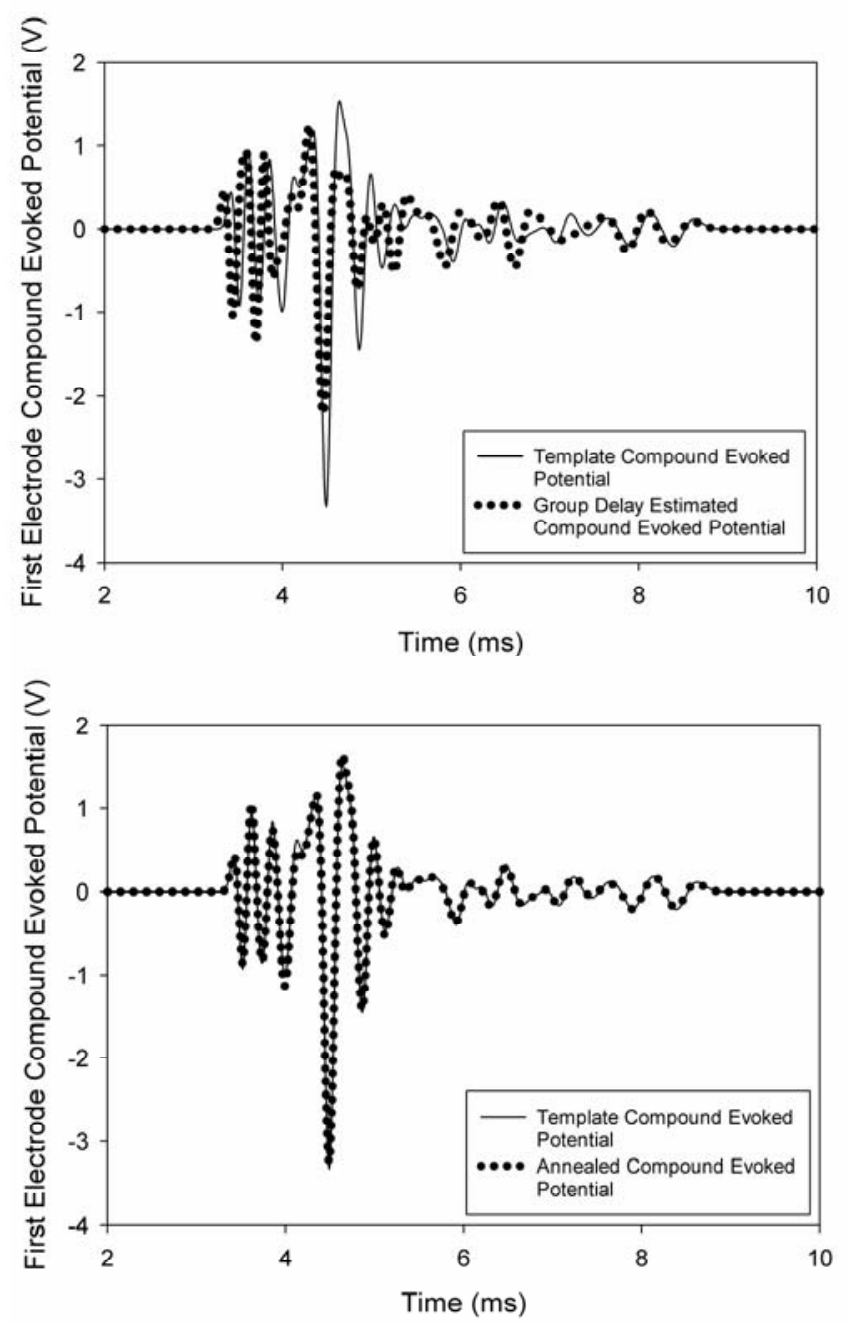

Figure 6. Graphs comparing the template maximal compound evoked potential to the group delay estimated maximal compound evoked potential and the annealed maximal compound evoked potential. The graph on the top shows the template maximal compound evoked potential at the first recording site $\Psi^{(1)}(t)$ and the group delay estimated maximal compound evoked potential at the first recording site $\widetilde{\Psi}^{(1)}(t)$. A comparison of $\Psi^{(1)}(t)$ to the annealed maximal compound evoked potential $\widehat{\Psi}^{(1)}(t)$ that results from optimization of the group delay estimate with the simulated annealing algorithm is shown in the graph on the bottom. The normalized final error between $\Psi^{(1)}(t)$ and $\widehat{\Psi}^{(1)}(t)$ as per (13), is $F E=7.673 \%$.

The effect of noise variations in the distance between the individual nerve fibers at the two virtual recording sites was also studied. Table 2 consists of typical Chi Squared Test results comparing the optimized and template histograms over a range of SNR values where the signal value is taken as the mean distance $\bar{r}$ and the noise power is the variance 
$\rho_{k}^{2}$ in the normally distributed distance variation around the mean value.

\begin{tabular}{|c|c|c|c|}
\hline $\begin{array}{l}\text { SNR } \\
(\mathrm{dB})\end{array}$ & $\begin{array}{c}\text { Group Delay } \\
\text { and Template } \\
\text { Distribution } \\
Q\left(\chi^{2} \mid x\right)\end{array}$ & $\begin{array}{l}\text { Annealed and } \\
\text { Template } \\
\text { Distribution } \\
Q\left(\chi^{2} \mid x\right)\end{array}$ & $\begin{array}{c}\text { Annealed } \\
\text { and } \\
\text { Template } \\
\text { Transient } \\
\text { Final Error } \\
F E(\%)\end{array}$ \\
\hline 0 & 0.3958 & 0.7235 & 20.93 \\
\hline 20 & 0.7101 & 0.9912 & 7.673 \\
\hline 40 & 0.7753 & 0.8850 & 10.54 \\
\hline
\end{tabular}

Table 2. Chi Square Test results and final normalized error $F E$ for different values of SNR associated with normally distributed random variations in the perpendicular distance between the recording electrode site and the concomitant nerve fiber.

The final error in percent, as shown in Table 2, was calculated as per (13).

$$
F E=\left(\frac{\left\|\Psi^{(1)}(t)-\widehat{\Psi}^{(1)}(t)\right\|_{2}}{\left\|\Psi^{(1)}(t)\right\|_{2}}\right) 100 \%
$$

\section{DISCUSSION}

The results of the simulation study shown earlier demonstrate that the technique presented herein can, with reasonable accuracy, retrieve the conduction velocity distribution in the presence of noise introduced through variations in the perpendicular distance between the recording site and the contributing fiber. The algorithm is effective even in reasonably high noise situations where the SNR associated with variations in the nerve fiber depth is 0 $\mathrm{dB}$. In the $0 \mathrm{~dB}$ case, a Chi Square Test comparing the actual template nerve fiber size distribution $\boldsymbol{d}$ with the distribution obtained from the group delay extraction $\widetilde{\boldsymbol{d}}$ yields a result of 0.3958 . After application of the simulated annealing algorithm to yield the optimized distribution $\widehat{\boldsymbol{d}}$, a Chi Square Test comparing the optimized distribution to the actual template distribution $\boldsymbol{d}$ yielded a higher value of 0.7235 which suggests that the annealed diameter set and the template set are more consistent with a single distribution than the template set and the group delay set. Similar results were given in Table 2, for higher SNR values associated with variation in the perpendicular nerve fiber and recording site distance.

The graphs shown in Figure 6 further demonstrate the ability of the simulated annealing optimization approach to improve upon the maximal compound evoked potential $\widetilde{\Psi}^{(1)}(t)$ estimated using the group delay extracted population $\widetilde{\boldsymbol{d}}$ as compared to the template maximal compound evoked potential $\Psi^{(1)}(t)$. After application of the simulated annealing algorithm, the bottom graph in Figure 6 demonstrates a reasonable convergence between the template maximal compound evoked potential $\Psi^{(1)}(t)$ and the maximal compound evoked potential $\widehat{\Psi}^{(1)}(t)$ associated with the optimized fiber diameter population $\widehat{\boldsymbol{d}}$. This convergence is further demonstrated by the relatively low normalized final error of $7.673 \%$.

Since the optimization process is random in nature, a successive decrease in the normalized final error is not always observed with increasing SNR values as demonstrated between the $20 \mathrm{~dB}$ and $40 \mathrm{~dB}$ data shown in Table 2. The increase in the normalized final error from the $20 \mathrm{~dB}$ to the $40 \mathrm{~dB}$ case is consistent with a lower value of the Chi Square Test comparing the template distribution to the annealed distribution for an SNR value of $40 \mathrm{~dB}$ than for the $20 \mathrm{~dB}$ case.

The proposed technique for measuring the size distribution of nerve fibers that contribute to the maximal compound evoked potential has several advantages over other earlier proposed methods. Unlike some previous techniques $[5 ; 10]$, no inherent assumptions are made regarding size based classification of contributing single fiber evoked potentials. Each contributing single fiber evoked potential can, in theory, have a unique wave shape. The fact that many of the other techniques stipulate specific forms of the single fiber action potential waveforms based upon dividing the range of fiber diameters into distinct groups, makes direct comparison of these techniques problematic.

One of the disadvantages of the proposed approach, in comparison to other techniques is the necessity to perform a series of compound evoked potential measurements associated with a train of successively increasing stimulus current pulse amplitudes. While the measurement associated with the proposed method is more involved, the protocols for extracting individual contributing evoked potentials based upon a successively increasing stimulus pulse amplitude is well established in the literature on motor unit number estimation [13].

\section{REFERENCES}

[1] P. A. Parker and P. Kelly, "Nerve conduction velocity measurement techniques," Journal of Clinical Engineering, vol. 7, no. 2, pp. 153158, Apr.1982.

[2] I. Atroshi, C. Gummesson, R. Johnsson, and E. Ornstein, "Diagnostic properties of nerve conduction tests in population-based carpal tunnel syndrome," BMC Musculoskeletal Disorders, vol. 4, no. 9 May2003.

[3] Y. Harati, "Diabetic peripheral neuropathies," Annals of Internal Medicine, vol. 107, pp. 546-559, 1987.

[4] L. J. Dorfman, K. L. Cummins, G. M. Reaven, J. Ceranski, M. S. Greenfield, and L. Doberne, "Studies of diabetic polyneuropathy using conduction velocity distribution (DCV) analysis," Neurology, vol. 33, no. 6 , pp. 773-779, June1983.

[5] K. L. Cummins, L. J. Dorfman, and D. H. Perkel, "Nerve fiber conduction-velocity distributions, II Estimation based on two compound action potentials," Electro-Encephal. Clin. Neurophysiol, vol. 46, no. 6 , pp. 647-658, 1979.

[6] L. J. Dorfman, "The distribution of conduction velocities (DCV) in peripheral nerves: a review," Muscle \& Nerve, vol. 7, no. 1, pp. 2-11, 1984.

[7] J. A. Gonzalez-Cueto and P. A. Parker, "Deconvolution estimation of nerve conduction velocity distribution," IEEE Transactions on Biomedical Engineering, vol. 49, no. 2, pp. 140-151, Feb.2002.

[8] F. A. Papadopoulou and S. M. Panas, "Estimation of the nerve conduction velocity distribution by peeling sampled compound action potentials," IEEE Transactions on Magnetics, vol. 35, no. 3, pp. 1801-1804, May1999. 
[9] D. Gu, R. E. Gander, and E. C. Crichlow, "Determination of nerve conduction velocity distribution from sampled compound action potential signals," IEEE Transactions on Biomedical Engineering, vol. 43, no. 8, pp. 829-838, Aug.1996.

[10] Y. X. Tu, A. Wernsdorfer, S. Honda, and Y. Tomita, "Estimation of Conduction velocity distribution by regularized-least-squares method," IEEE Transactions on Biomedical Engineering, vol. 44, no. 11, pp. 1102-1106, Nov.1997.

[11] R. B. Szlavik, "A novel method for characterization of peripheral nerve fiber size distributions by group delay," IEEE Transactions on Biomedical Engineering, 2008, accepted.

[12] S. M. Fleisher, M. Studer, and G. S. Moschytz, "Mathematical model of the single-fiber action potential," Medical and Biological Engineering and Computing, vol. 22, pp. 433-439, 1984.

[13] A. J. McComas, P. R. W. Fawcett, M. J. Campbell, and R. E. P. Sica, "Electrophysiological estimation of the number of motor units within a human muscle," Journal of Neurology, Neurosurgery and Psychiatry, vol. 34, pp. 121-131, 1971.

[14] I. A. Boyd and M. R. Davey, Composition of Peripheral Nerves. Edinburgh: E \& S Livingstone Ltd., 1968.

[15] R. B. Szlavik and H. de Bruin, "Simulating the distribution of axon size in nerves," Canadian Medical and Biological Engineering Society, 1997, pp. 168-169.

[16] N. Metropolis, A. W. Rosenbluth, M. N. Rosenbluth, A. H. Teller, and E. Teller, "Equation of state calculations by fast computing machines," The Journal of Chemical Physics, vol. 21, no. 6, pp. 10871092, June1953.

[17] E. Aarts and J. Korst, Simulated Annealing and Boltzmann Machines John Wiley \& Sons, 1989.

[18] W. H. Press, B. P. Flannery, S. A. Teukolsky, and W. T. Vetterling, Numerical Recipes (Fortran). New York: Cambridge University Press, 1990. 